\title{
On designing state feedback controller for time-delay system of neutral type
}

\author{
Zhang Zhan', Wei Shaoqing ${ }^{2}$, Zhang Jianhua ${ }^{2}$, Ma Jia ${ }^{2}$,Li Mingyu ${ }^{2}$ \\ ${ }^{1}$ China Three Gorges University, College of Electrical Engineering and New Energy, Hubei, China \\ ${ }^{2}$ Hebei University of Science and Technology, School of Electrical Engineering and Information, China \\ zhan.zhang1986@gmail.com, wei_shaoqing@163.com
}

\begin{abstract}
This paper makes the linear uncertain neutral system as the research object, and designs state feedback observer, achieving the observed state. And the paper designs an observer based on state feedback controller, to control the neutral system. And then we make simulation through Matlab Simulink toolbox, verifying the effectiveness of the algorithm. In addition, by using cone complementarity algorithm, the solutions to the observer gain matrices are obtained by solving a set of linear matrix inequalities (LMIs).

Index Terms - neutral system, the feedback controller, linear matrix inequality (LMI)

\section{Introduction}

In recent decades, the stability problems of time-delay neutral system have been brought great attention by many scholars at home and abroad, especially the analysis of feedback controlling about system. And the feedback controlling has become one of the very active researches in automatic controlling [1-3]. In the past two years, the feedback controlling delay system is used as separate chapters in controlling theory monograph by writers [4-6].

Neutral time-delay system is a special time-delay system; it can accurately reflect the laws of things' changes, revealing the nature of things. [7] discussed a problem of a varying delay neutral system of delay-dependent asymptotic stability. [8] studied a class of problem of Lurie controlling systems with time-varying asymptotic stability and $\mathrm{H} \infty$ state feedback controller, by using Lyapunov function and linear matrix inequalities. This article places uncertain neutral system as the research object, and gives the designed method of the state feedback observer, then designs the controller based on the observer, controlling the neutral system and using Matlab simulation to verify its feasibility.
\end{abstract}

\section{Description of the system}

Establish the following Linear Uncertain Neutral Systems:

$$
\left\{\begin{array}{c}
\dot{x}(t)=(A+\Delta A) x(t)+A_{h} x(t-h) \\
+A_{d} \dot{x}(t-h)+B u(t) \\
y(t)=C x(t) \\
x(t)=\phi(t)
\end{array}\right.
$$

\footnotetext{
* This work is partially supported by NSF Grant \#2003168 to H. Simpson and CNSF Grant \#9972988 to M. King.
} 


$$
\begin{aligned}
\dot{e}(t) & =(A+\Delta A-L C) e(t) \\
& +\left(A_{h}-L_{h} C\right) e(t-h)+A_{d} \dot{e}(t-h)
\end{aligned}
$$

For the error system (3)

$$
\frac{d}{d t}\left\{\dot{e}(t)-A_{d} \dot{e}(t-h)\right\}=\bar{A} e(t)+\overline{A_{h}} e(t-h)
$$

Which $\bar{A}=A+\Delta A-L C \quad \overline{A_{h}}=A_{h}-L_{h} C$

\section{Main conclusions and certification}

Theorem 1 If there is a positive definite matrix $\mathrm{R}, \mathrm{Q}$ and rectangular matrices $\mathrm{W}$, so that the following LMIs are established

$$
\begin{aligned}
& {\left[\begin{array}{ccc}
\Sigma & R A_{h}-W_{h} C-A^{T} R A_{d}+C^{T} W^{T} R A_{d} & \sqrt{2} R D \\
* & -Q & 0 \\
* & 0 & -I
\end{array}\right]<0} \\
& Q+A_{d}^{T} E^{T} E A_{d}-A_{h}^{T} R A_{d}+C^{T} W_{h}^{T} A_{d} \\
& -A_{d}^{T} R A_{h}+A_{d}^{T} W_{h} C>0
\end{aligned}
$$

Where

$$
\begin{aligned}
\Sigma & =Q+A_{d}^{T} E^{T} E A_{d}-A_{h}^{T} R A_{d}-A_{d}^{T} R A_{h}+C^{T} W_{h}^{T} A_{d} \\
& +A_{d}^{T} W_{h} C+E^{T} E+A^{T} R+R A-C^{T} W^{T}-W C
\end{aligned}
$$

The error system (3) is asymptotically stable, and the observer gain matrix is the following

$$
L=R^{-1} W \quad L_{h}=R^{-1} W_{h}
$$

Proof: Define g function:

$$
g\left(e_{t}\right)=e(t)-A_{d} e(t-h)
$$

Take

$$
V_{1}\left(e_{t}\right)=g^{T}\left(e_{t}\right) \operatorname{Rg}\left(e_{t}\right)
$$

$V_{2}\left(e_{t}\right)=\int_{t-h}^{t} e(s) d s$

First, in order to guarantee the positive definiteness of $\mathrm{V}$ requires $\mathrm{R}>0$,

$$
Q+A_{d}^{T} E E A_{d}-\bar{A}_{h}^{T} R A_{d}-A_{d}^{T} R \bar{A}_{h}>0
$$

Taking the derivative of $\mathrm{V}$ under the conditions of the error system formula (4),

$\dot{V}_{1}=\dot{g}^{T}\left(e_{t}\right) \operatorname{Rg}\left(e_{t}\right)+g^{T}\left(e_{t}\right) \operatorname{Rg}\left(e_{t}\right)$

$$
\begin{aligned}
& =\left[\bar{A} e(t)+\overline{A_{h}} e(t-h)\right]^{T} R\left[e(t)-A_{d} e(t-h)\right] \\
& +\left[e(t)-A_{d} e(t-h)\right]^{T} R\left[\bar{A} e(t)+\overline{A_{h}} e(t-h)\right] \\
& =e^{T}(t)\left(\bar{A}^{T} R+R \bar{A}\right) e(t)+2 e^{T}(t)\left(\begin{array}{l}
R \overline{A_{h}}- \\
\bar{A}^{T} R A_{d}
\end{array}\right) e(t-h) \\
& -e^{T}(t-h)\left[{\overline{A_{h}}}^{T} R A_{d}+A_{d}^{T} R \overline{A_{h}}\right] e(t-h) \\
& =e^{T}(t)\left[(A+\Delta A-L C)^{T} R+R(A+\Delta A-L C)\right] e(t) \\
& +2 e^{T}(t)\left[\left(R \overline{A_{h}}-(A+\Delta A-L C)^{T} R A_{d}\right)\right] e(t-\mathfrak{h}) \\
& -e^{T}(t-h)\left[{\overline{A_{h}}}^{T} R A_{d}+A_{d}^{T} R \overline{A_{h}}\right] e(t-h) \\
& =e^{T}(t)\left[\begin{array}{l}
\Delta A^{T} R+A^{T} R+R A+R \Delta A- \\
C^{T} L^{T} R-R L C
\end{array}\right] e(t) \\
& +2 e^{T}(t)\left[\begin{array}{l}
R \overline{A_{h}}-\Delta A^{T} R A_{d}-A^{T} R A_{d} \\
+C^{T} L^{T} R A_{d}
\end{array}\right] e(t-h) \\
& -e^{T}(t-h)\left[{\overline{A_{h}}}^{T} R A_{d}+A_{d}^{T} R \overline{A_{h}}\right] e(t-h) \\
& =2 e^{T}(t) R \Delta A e(t)+e^{T}(t)\left(\begin{array}{l}
A^{T} R+R A- \\
C^{T} L^{T} R-R L C
\end{array}\right) e(t) \\
& +2 e^{T}(t)\left[R \overline{A_{h}}-A^{T} R A_{d}+C^{T} L^{T} R A_{d}\right] e(t-h) \\
& -2 e^{T}(t) R \Delta A A_{d} e(t-h) \\
& -e^{T}(t-h)\left[{\overline{A_{h}}}^{T} R A_{d}+A_{d}^{T} R \overline{A_{h}}\right] e(t-h) \\
& \leq e^{T}(t) R D F(t) F^{T}(t) D^{T} \operatorname{Re}(t)+e^{T}(t) E^{T} E e(t) \\
& +e^{T}(t)\left(A^{T} R+R A-C^{T} L^{T} R-R L C\right) e(t)+ \\
& 2 e^{T}(t)\left[R \overline{A_{h}}-A^{T} R A_{d}+C^{T} L^{T} R A_{d}\right] e(t-h) \\
& +e^{T}(t) R D F(t) F^{T}(t) D^{T} \operatorname{Re}(t)+ \\
& e^{T}(t-h) A_{d}^{T} E^{T} E A_{d} e(t-h) \\
& -e^{T}(t-h)\left[{\overline{A_{h}}}^{T} R A_{d}+A_{d}^{T} R \overline{A_{h}}\right] e(t-h) \\
& \leq e^{T}(t)\left(\begin{array}{c}
2 R D D^{T} R+E^{T} E+A^{T} R \\
+R A-C^{T} L^{T} R-R L C
\end{array}\right) e(t) \\
& +e^{T}(t)\left[\begin{array}{l}
R \overline{A_{h}}-A^{T} R A_{d} \\
+C^{T} L^{T} R A_{d}
\end{array}\right] Q^{-1}\left[\begin{array}{l}
R \overline{A_{h}}-A^{T} R A_{d} \\
+C^{T} L^{T} R A_{d}
\end{array}\right]^{T} e(t) \\
& +e^{T}(t-h)\left(Q+A_{d}^{T} E^{T} E A_{d}-{\overline{A_{h}}}^{T} R A_{d}\right. \\
& \left.-A_{d}^{T} R \overline{A_{h}}\right) e(t-h)
\end{aligned}
$$




$$
\begin{aligned}
\dot{V}_{2} & =e^{T}(t)\left[Q+A_{d}^{T} E^{T} E A_{d}-\bar{A}_{h}^{T} R A_{d}-A_{d}^{T} R \bar{A}_{h}\right) e(t) \\
& -e^{T}(t-h)\left(Q+A_{d}^{T} E^{T} E A_{d}-\bar{A}_{h}^{T} R A_{d}-A_{d}^{T} R \bar{A}_{h}\right) e(t-\mathfrak{h})
\end{aligned}
$$

Therefore

$$
\dot{V}\left(e_{t}\right) \leq e^{T}(t) \Omega e(t)
$$

Where

$$
\begin{aligned}
\Omega & =A^{T} R+R A+E^{T} E+2 R D D^{T} R+Q+A_{d}^{T} E^{T} E A_{d} \\
& -\bar{A}_{h}^{T} R A_{d}-A_{d}^{T} R \bar{A}_{h}-C^{T} L^{T} R-R L C \\
& +\left(\begin{array}{l}
R \overline{A_{h}}-A^{T} R A_{d} \\
+C^{T} L^{T} R A_{d}
\end{array}\right) Q^{-1}\left(\begin{array}{l}
R \overline{A_{h}}-A^{T} R A_{d} \\
+C^{T} L^{T} R A_{d}
\end{array}\right)^{T}
\end{aligned}
$$

By schur complement, it is equivalent to the following form:

$$
\left[\begin{array}{ccc}
\Theta & R \overline{A_{h}}-\bar{A}^{T} R A_{d}+C^{T} L^{T} R A_{d} & \sqrt{2} R D \\
* & -Q & 0 \\
* & 0 & -I
\end{array}\right]<0
$$

Where

$$
\begin{aligned}
\Theta & =A^{T} R+R A+E^{T} E+Q+A_{d}^{T} E^{T} E A_{d} \\
& -\bar{A}_{h}^{T} R A_{d}-A_{d}^{T} R \bar{A}_{h}-C^{T} L^{T} R-R L C
\end{aligned}
$$

After a simple deformation matrix inequality, (10) is equivalent to the linear matrix inequalities (5).And the inequality (9) is equivalent to the following inequality

$$
\begin{aligned}
& Q+A_{d}^{T} E E A_{d}-\left(A_{h}-L_{h} C\right)^{T} R A_{d} \\
& -A_{d}^{T} R\left(A_{h}-L_{h} C\right)>0
\end{aligned}
$$

That is

$$
\begin{aligned}
& Q+A_{d}^{T} E E A_{d}-A_{h}^{T} R A_{d} A_{d}-A_{d}^{T} R A_{h} \\
& +C^{T} W_{h}^{T} A_{d}+A_{d}^{T} W_{h} C>0
\end{aligned}
$$

Where $\mathrm{W}=\mathrm{RL}$, theorem is proved.

\section{Simulation}

Base on the theorem 1 given following simulation examples, it verifies that the criterion in Theorem 1 is feasible.

The example 1 Neutral system can be selected as

$$
\dot{x}(t)-C \dot{x}(t-\tau)=A x(t)+A_{1} x(t-h(t))
$$

Where

$$
A=\left[\begin{array}{ll}
2 & 1 \\
1 & 3
\end{array}\right] \quad A_{1}=\left[\begin{array}{ll}
2 & 1 \\
2 & 1
\end{array}\right] \quad C=\left[\begin{array}{ll}
6 & 0 \\
0 & 5
\end{array}\right]
$$

Thus by the contents of the Theorem 1, we obtain

$$
Q=\left[\begin{array}{cc}
45.7416 & 1.4751 \\
1.4751 & 51.8909
\end{array}\right] R=\left[\begin{array}{cc}
45.2452 & 0.3747 \\
0.3747 & 25.6752
\end{array}\right]
$$

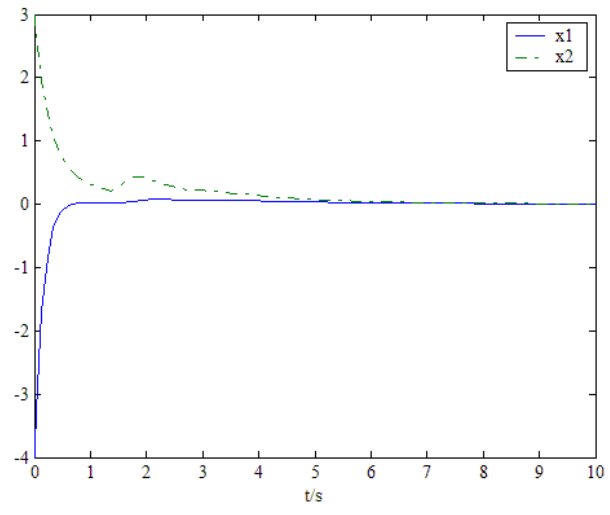

Figure 1 The simulation curves of the state of the system

Figure 1 illustrates the system state will soon be able to reach a steady state, so the criterion of theorem 1 is feasible. That is, by using the effect of observer and controller in theorem1, we can control the system, making itself from unstable to stable.

\section{Summary}

In this paper, we use uncertain neutral system as the research object, and give design of observer of the neutral system. And then we design controller by observer, finally control the system. All conclusions are given in the form of the LMI, and we simulate through Matlab Simulink toolbox, to verify the effectiveness of the algorithm.

\section{References}

[1] Fridman E. New Liapunov-Kr asovskii functionals for stability of linear retarded and neutral type systems [J] .Systems Control Letter, 2001, 43(4): 309-319.

[2] Han Q L. Robust stability of uncertain delay-differential systems o f neutral type [J]. Automatica, 2002, 38(4):719-723.

[3] Zhang YP, Liu XZ, Zhu H, Zhong SM. Stability analysis and control synthesis for a class of switched neutral systems. Appl Math Comput.2007; 190(2):58 - 66.

[4] Yu K W, Lien C H.Stability criteria for uncertain neutral systems with interval time-varying delays $[\mathrm{J}]$.Chaos, Solutions \& Franctals, 2008, 28: 650-657.

[5] Cong S, Qian W, Fei SM. On exponential stability of switched systems with delay: multiple Lyapunov functions approach. Proceedings of the 26th chinese control conference, Zhangjiajie, China: 2007. pp. 664 - 668.

[6] He Y, Wu M, She J H, et al. Delay-dependent robust stability criteria for uncertain neutral systems with mixed delays [J].Sys Control Lett, 2004, 51: 57-65.

[7] Guocai Liu. Peijun Ju. Delay-dependent Asymptotical Stability for Neutral System with Time-varying Delays; Journal of Chongqing Normal University (Natural Science) 2011, 39:22-26

[8] Chong Fan ,Jundong Bao .Design of $\mathrm{H}_{-} \infty$ State Feedback Controller for Lurie Control Systems with Time-Varying Delay ;Journal of Inner Mongolia Normal University(Natural Science Edition .2010, 28:48-51

[9] Tian J K, Zhong S M, Xiong L L. Delay dependent absolute stability of Lurie control systems with multiple time delays [J]. Applied Mathematics and Computation, 2007, 188:379-384.

[10] Chen Zhaona, Zheng Yan, Jing Yuanwei. Design of Multi-objective Fuzzy Dynamic Output Feedback Controller. Journal of Northeastern University .2010,03 